\title{
Turbulence Suppression by Energetic Particle Effects in Modern Optimized Stellarators
}

\author{
A. Di Siena $\oplus^{1,2, * \dagger}$ A. Bañón Navarro $\oplus^{2, *, *}$ and F. Jenko $\circledast^{2, \S}$ \\ ${ }^{1}$ The University of Texas at Austin, 201 East 24th Street, Austin, Texas 78712, USA \\ ${ }^{2}$ Max Planck Institute for Plasma Physics, Boltzmannstrasse 2, 85748 Garching, Germany
}

(Received 29 February 2020; revised 17 July 2020; accepted 30 July 2020; published 3 September 2020)

\begin{abstract}
Turbulent transport is known to limit the plasma confinement of present-day optimized stellarators. To address this issue, a novel method to strongly suppress turbulence in such devices is proposed, namely the resonant wave-particle interaction of suprathermal particles-e.g., from ion-cyclotron-resonancefrequency heating - with turbulence-driving microinstabilities like ion-temperature-gradient modes. The effectiveness of this mechanism is demonstrated via large-scale gyrokinetic simulations, revealing an overall turbulence reduction by up to $65 \%$ in the case under consideration. Comparisons with a tokamak configuration highlight the critical role played by the magnetic geometry and the first steps into the optimization of fast particle effects in stellarator devices are discussed. These results hold the promise of new and still unexplored stellarator scenarios with reduced turbulent transport, essential for achieving burning plasmas in future devices.
\end{abstract}

DOI: 10.1103/PhysRevLett.125.105002

Introduction.-Turbulent transport, generated by pressuregradient-driven microinstabilities and inducing significant energy and particle losses, is often a key limiting factor for the performance of magnetic confinement fusion devices. Historically, most investigations on plasma turbulence were carried out for tokamak experiments, while stellarators were usually limited by neoclassical (collisional) transport. However, the recent effort spent in the design of modern stellarators - with reduced neoclassical losses and thus improved confinement performances-raised the role of turbulent transport as the dominant mechanism of confinement degradation also for stellarator devices [1-5]. In this context, we note recent observations at Wendelstein 7-X (W7-X) [6,7], which show that ion-temperature-gradient (ITG) [8] driven turbulent transport clearly exceeds its neoclassical counterpart $[9,10]$. This emphasizes the great importance of identifying mechanisms able to suppress turbulent transport in modern optimized stellarators systematically.

In the present Letter, we propose a novel method to strongly suppress turbulence in such devices. It is based on a wave-particle resonant interaction between suprathermal (fast) ions and ITG microinstabilities, previously identified for tokamak plasmas in both experiments [11] and simulations $[12,13]$. The beneficial role of this mechanism is

Published by the American Physical Society under the terms of the Creative Commons Attribution 4.0 International license. Further distribution of this work must maintain attribution to the author(s) and the published article's title, journal citation, and DOI. Open access publication funded by the Max Planck Society. demonstrated for the first time in stellarators via nonlinear gyrokinetic simulations [14] of W7-X. We show that fast ions - under appropriate conditions - significantly impact turbulence-induced energy losses, potentially leading to a considerable reduction of turbulent transport. This mechanism requires (i) steep temperature and rather flat density profiles and (ii) moderate fast-particle temperatures. Such conditions can be achieved by ioncyclotron-resonance-frequency (ICRF) heating schemes [15]. However, due to the subdominant role played by the anomalous transport in previous stellarator experiments, no evidence of turbulence suppression by ICRF heating has been documented in such devices. For this reason, these findings are attractive for optimized stellarators, particularly for W7-X, whose ICRF system is currently under construction [16], providing an external tool—such as suprathermal particles-to continuously reduce turbulent transport.

The critical role played by the magnetic geometry in the dynamics of this resonance effect is also investigated, revealing a key difference behavior between tokamaks and stellarators. We show that the optimal fast-particle temperature, which maximizes the resonant stabilization, is affected by the different resonant constraints imposed by each stellarator field line. We explore the large number of degrees of freedom in changing the magnetic geometry available in stellarators and identify artificial configurations enhancing the fast-particle turbulence suppression, thus suggesting new pathways for stellarator optimization in the presence of fast particles.

Model description.-The wave-particle resonant interaction [12,13] we propose to exploit in optimized stellarators occurs whenever the frequency of the most 
unstable mode $\omega_{k}$ (with positive values denoting modes propagating in the ion diamagnetic direction) matches the binormal drift frequency of the (normalized) energetic particles $\omega_{d, f}$, i.e.,

$$
\omega_{k}=\omega_{d, f}=-\frac{k_{y} \mathcal{K}_{y} T_{f} E_{f}}{q_{f} B_{0}} .
$$

Here, $T_{f}$ and $q_{f}$ are the energetic particle temperature and charge (normalized to the electron temperature and elementary charge), $B_{0}$ the background magnetic field (normalized to its value on axis), $k_{y}$ the (positive) binormal wave vector of the selected mode [normalized to the thermal gyroradius $\rho_{s}=\left(T_{e} / m_{i}\right)^{1 / 2} / \Omega_{i}$, with $\Omega_{i}=\left(q_{i} B_{0}\right) /\left(m_{i} c\right)$ the gyrofrequency, $m_{i}$ the main ion mass, $q_{i}$ the charge], and

$$
\mathcal{K}_{y}=-\frac{\left(\mathbf{B}_{0} \times \nabla B_{0}\right) \cdot \hat{y}}{B_{0}^{2}}
$$

is the binormal curvature term, where $\hat{y}$ denotes the unit vector along the binormal direction. Moreover, $E_{f}=\left(2 v_{\|}^{2}+\mu B_{0}\right)$, with $v_{\|}$the velocity component parallel to the background magnetic field [normalized to $v_{\text {th }, f}=\left(2 T_{f} / m_{f}\right)^{1 / 2}$, with $m_{f}$ the fast ion mass] and $\mu$ the magnetic moment (normalized to $T_{f} / B_{0}$ ).

As a direct consequence of Eq. (1), the term $\mathcal{K}_{y}$ must be negative for modes propagating in the ion diamagnetic direction-such as ITG modes-to fulfill the resonance condition. The direction of the resonant energy exchanged is set by the radial derivative of the fast ion distribution function (drive term) [13]. Negative (positive) values indicate that the energetic particles are taking (giving) energy from (to) the plasma microinstabilities with consequent damping (growth) of the mode. By assuming an equivalent Maxwellian distribution, the drive term reads as $a / L_{n, f}+a / L_{T, f}\left(v_{\|}^{2}+\mu B_{0}-3 / 2\right)$, with $a / L_{T, f}$ and $a / L_{n, f}$ being the fast ion normalized logarithmic temperature and density gradients and $a$ minor radius of the device. It leads to negative values within $v_{\|}^{2}+\mu B_{0}<3 / 2$ when $a / L_{T, f} \gg a / L_{n, f}$. Therefore, a negative (beneficial) fast-particle contribution to the ITG microinstability can be achieved only when (i) $a / L_{T, f} \gg a / L_{n, f}$ and (ii) $\omega_{k}$ matches $\omega_{d, f}$ in the phase-space region where $v_{\|}^{2}+\mu B_{0}<3 / 2$. These conditions are usually satisfied by energetic particles generated via ICRF heating [15].

Gyrokinetic simulations for optimized stellarators.The impact of this resonant mechanism on the turbulent transport of stellarators is investigated through numerical simulations of W7-X, performed with the gyrokinetic code GENE [5,17-19]. To identify stellarator-specific features, these findings are compared to those of a typical tokamak (ASDEX Upgrade). These two devices are conceptually different and require separate numerical setups. While the
TABLE I. Plasma parameters used in the simulations.

\begin{tabular}{lcccc}
\hline \hline Species & $T / T_{e}$ & $n / n_{e}$ & $a / L_{T}$ & $a / L_{n}$ \\
\hline $\mathrm{H}$ (fast) & $1.0-20.0$ & 0.06 & 18.0 & 0.0 \\
$\mathrm{D}$ (bulk) & 1.0 & 0.94 & 2.5 & 0.0 \\
\hline \hline
\end{tabular}

former is intrinsically axisymmetric and, hence, described by one representative flux tube, the latter requires to treat the entire flux surface to correctly capture the dynamics. For this reason, we present results obtained (i) by full flux-surface and (ii) by flux-tube simulations. The first approach captures correctly the nonaxisymmetric stellarator geometry, while the second one is valid for tokamaks since it follows only a single magnetic field line.

The chosen simulation parameters, summarized in Table I, are inspired by realistic W7-X data [10] and refer to experimental conditions in which the turbulence is driven by ITG modes. The bulk plasma is composed of deuterium (D) and electrons. The latter are assumed to have an adiabatic response. The suprathermal ions are modeled with ICRF parameters of a minority heating scenario of hydrogen $(\mathrm{H})$ in $\mathrm{D}$ with $n_{\mathrm{H}} / n_{e}=6 \%$, as recently proposed in Ref. [16] for the ICRF system in W7-X (currently under construction). Plasma quasineutrality is always fulfilled. Moreover, we consider $a / L_{T f}=18$, which is consistent with the realistic values observed in ICRF H-minority heating in tokamak experiments [11,20]. The hydrogen minority is modeled with an equivalent Maxwellian background. Based on previous tokamak studies using more realistic distribution functions [21], this choice is not expected to change qualitatively our results. A more detailed analysis quantifying this effect in stellarators is left for future studies. The high-mirror configuration [10] is employed for W7-X, while an ASDEX Upgrade geometry [22] — with safety factor $q=1.34$ and magnetic shear $\hat{s}=0.44$-is used for the tokamak simulations. Finally, the grid resolution in radial, binormal and parallel to the magnetic field-line directions for the full surface and flux-tube GENE simulations are, respectively, $(x, y, z)=(144,128,192)$ and $(x, y, z)=(250,32,128)$. A fixed resolution in the magnetic moment and parallel velocity $\left(\mu, v_{\|}\right)=(20,32)$ is used.

Turbulence reduction through fast particles. - As a first attempt to quantify the impact of the wave-particle resonance mechanism in W7-X (and similar optimized stellarators), the nonlinear energy fluxes are studied for different energetic particle temperatures and are compared to those obtained for ASDEX Upgrade. The magnetic geometry is kept constant throughout this analysis. The flux-surface averaged bulk ion heat fluxes-normalized to $Q_{\mathrm{gB}}=n_{i} T_{i} c_{s}\left(\rho_{s} / a\right)^{2}$-are shown in Fig. 1. Here, $c_{s}=\left(T_{e} / m_{i}\right)^{1 / 2}$ represents the sound speed. By looking at Fig. 1, we note a particularly strong dependence of the main ion fluxes on the externally heated $\mathrm{H}$ temperature for 


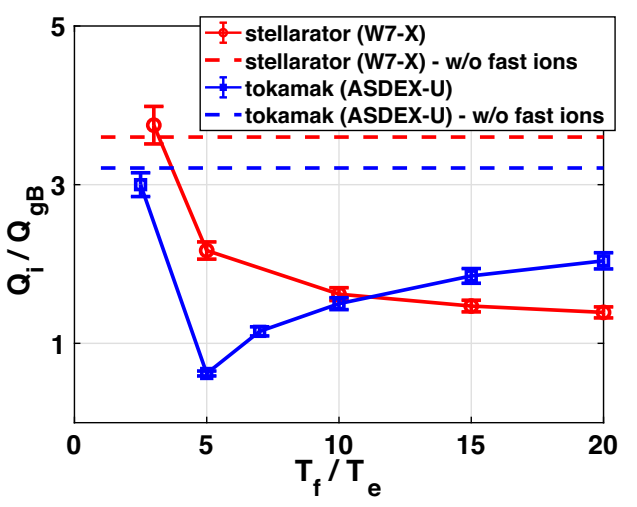

FIG. 1. Nonlinear main ion heat fluxes in GyroBohm $\left(Q_{\mathrm{gB}}\right)$ units for different energetic particle temperatures $T_{f} / T_{e}$. The horizontal dotted lines denote the fluxes obtained without fast ions (w/o denotes without).

both devices. However, while the tokamak exhibits a shard drop at $T_{f} / T_{e}=5$ and an increase for larger temperatures (as in Ref. [13]), a pronounced monotonic reduction is found for W7-X. The latter approaches a broad plateau in the bulk ion energy fluxes in the large fast-particle temperature limit. Nevertheless, a similar turbulence suppression of $\sim 65 \%$ compared to the case with only thermal particles [dashed lines in Fig. 1], is measured for both tokamaks and stellarators. Out of the overall stabilization, $\sim 15 \%$ is attributed to the dilution of the thermal ion species. It is measured by comparing our results to the ones obtained in simulations where the fast ion drive is set to zero (i.e., $\omega_{T, f}=0$ ).

The critical role of the resonant interaction between ITG modes and suprathermal ions is investigated by analyzing the $k_{y} \rho_{s}$ spectra of the time-averaged ion heat fluxes in Fig. 2(a). Taking as reference spectra the one obtained without fast ions, we note that the peak of the main ion heat flux spectra exhibits a downshift toward smaller $k_{y} \rho_{s}$ as the energetic particle temperature increases. It is located at $k_{y} \rho_{s}=0.5$ for the cases without fast ions and moves to $k_{y} \rho_{s}=0.28$ at $T_{f}=15 T_{e}$. The energetic ion turbulent spectra, on the other hand, reveals negative turbulent
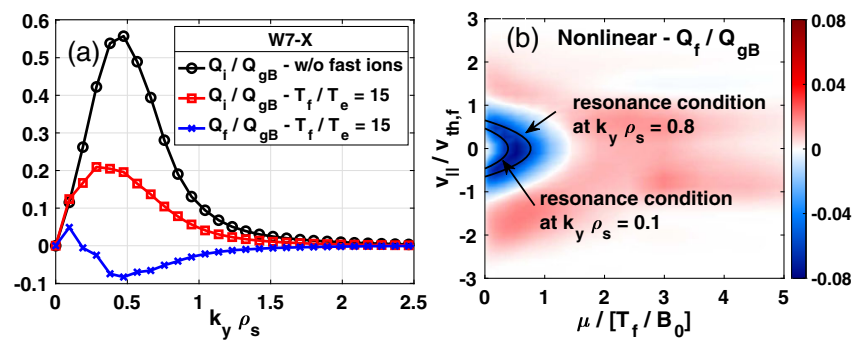

FIG. 2. Nonlinear saturated (a) main and energetic ion heat flux spectra and (b) velocity space structure of $Q_{f} / Q_{\mathrm{gB}}$ averaged over $k_{x}, k_{y}$, and $z$ at $T_{f} / T_{e}=15$ (w/o denotes without). The black contour lines in (b) indicate the phase-space resonance positions $\omega_{k}=\omega_{d, f}$ for $z=0$. energy fluxes, particularly pronounced at the wave vectors corresponding to the strongest ITG stabilization, i.e., $k_{y} \rho_{s}=0.5$. These findings are consistent with the theoretical predictions of the wave-particle mechanism. In particular, at a fixed temperature, each scale undergoes a different stabilization due to fast ion effects, significantly affecting the shape of the binormal heat flux spectra.

To further corroborate the theory, the velocity space structure $\left(v_{\|}, \mu\right)$ of $Q_{f} / Q_{\mathrm{gB}}$-averaged over the fieldaligned coordinate $z$-is illustrated in Fig. 2(b) for $T_{f} / T_{e}=15$. The black lines of Fig. 2(b) indicate the $\left(v_{\|}, \mu\right)$ values that satisfy the resonance conditions for the modes $0.1<k_{y} \rho_{s}<0.8$, i.e., corresponding to the strongest fast ion stabilizing effect observed in Fig. 2(a). Within these lines, we note the predominantly negative velocity phase-space structure of $Q_{f} / Q_{\mathrm{gB}}$, revealing an energy redistribution from the thermal ion driven ITG turbulence to the energetic particle species. This shows that the fast ions act as an effective sink of energy at $T_{f} / T_{e}=15$, reducing the main ITG drive.

Field-line dependencies. - To explore how the stellarator field-line dependence affects the dynamics of the waveparticle resonance interaction, we perform flux-tube simulations of W7-X by selecting distinct field lines. More precisely, we investigate the main ion heat flux behavior with the hydrogen temperature for two different flux tubes centered around the outboard midplane of the so-called (i) bean-shaped and (ii) teardrop cross sections (the two separated by a toroidal angle of $\pi / 10$ ); see Ref. [23] for the poloidal cross sections. The results are displayed in Fig. 3(a). We note that the energetic particle temperature corresponding to the minimum of the bulk ion heat flux moves as the flux tube is changed. It goes from $T_{f} / T_{e} \approx 12.5$ for the bean-shaped to $T_{f} / T_{e}=7$ for the teardrop flux tubes. This peculiar dependence is associated with pronounced modifications in the geometrical term $\mathcal{K}_{y}$ with the field line, as shown in Fig. 3(b). The impact of these different $\mathcal{K}_{y}$ to the wave-particle resonance interaction is demonstrated in Fig. 4 by studying the $z-\mu$ space structure of $Q_{f} / Q_{\mathrm{gB}}$ at $T_{f} / T_{e}=10$ for the different field lines [see Fig. 4(a) for the bean shape and Fig. 4(b) for the

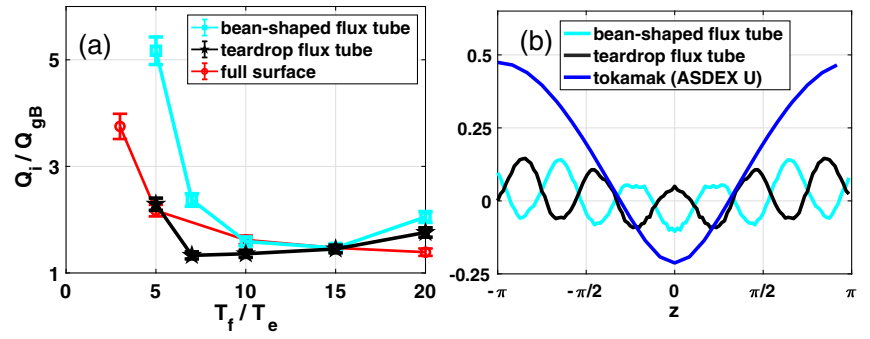

FIG. 3. Field-line dependence of the (a) nonlinear main ion heat fluxes for different energetic particle temperatures and field lines and (b) $\mathcal{K}_{y}$ versus $z$ for both tokamak and stellarators. 

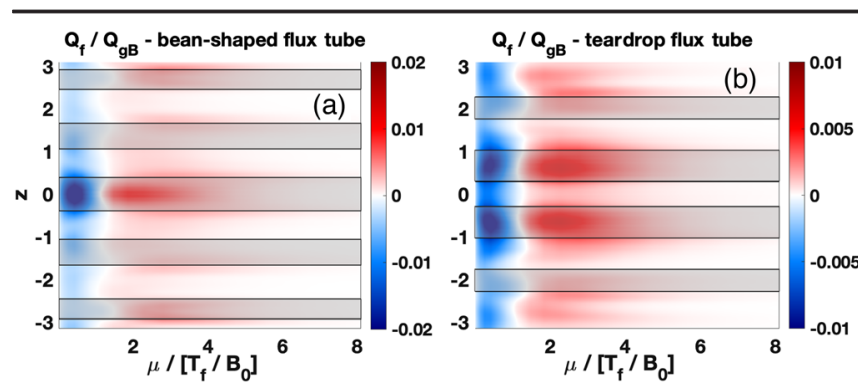

FIG. 4. Comparison of the $(z-\mu)$ structure of the binormal curvature term $\mathcal{K}_{y}$ and the $v_{\|}$averaged $Q_{f} / Q_{\mathrm{gB}}$ obtained from the bean-shaped and teardrop field lines at $T_{f} / T_{e}=10$. The area within the vertical gray boxes denotes the field-aligned values where $\mathcal{K}_{y}<0$.

teardrop]. By looking at these results, we observe a clear relation between $Q_{f} / Q_{\mathrm{gB}}$ and $\mathcal{K}_{y}$. In particular, $Q_{f} / Q_{\mathrm{gB}}$ exhibits the dominant negative contributions (suppression of ITG turbulence) when the resonance interaction becomes effective, namely when $\mathcal{K}_{y}<0$ (mainly at $z=0$ for the bean-shaped and $-1<z<1$ for the teardrop flux tubes). We also note that the overall negative region within $-1<z<1$ (which contributes the most to the resonance stabilization) of the teardrop flux tube is larger than the one observed for the bean-shaped case, having two negative peaks instead of one. As a consequence, the contribution of $\mathcal{K}_{y}$ in the energetic particle drift frequency $\left(\omega_{d, f}\right)$ is enhanced for the teardrop magnetic surface. Therefore, being $\omega_{k}$ only slightly affected by the changes in the field line (not shown here), $T_{f} / T_{e}$ must decrease to compensate the variation in $\mathcal{K}_{y}$ and match the ITG mode frequency [see Eq. (1)]. This interplay between $\mathcal{K}_{y}$ and $T_{f} / T_{e}$ moves the optimal fast-particle temperature for the teardrop flux tube to smaller values compared to the bean-shaped one. Ultimately, such a field-line behavior leads to the monotonic reduction observed in the full-surface simulations. A similar argument can explain why the differences between the field lines reduce as $T_{f} / T_{e}$ increases. At low temperatures, to match the slightly affected ITG mode frequency and compensate for the small contribution of $T_{f} / T_{e}$, the quantity $\mathcal{K}_{y} E_{f}$, entering the energetic particle drift frequency, must increase. This causes the differences in the structure of $\mathcal{K}_{y}$ to be more relevant. As the fast-particle temperature increases, the term $\mathcal{K}_{y} E_{f}$ must decrease, thus making the differences of $\mathcal{K}_{y}$ between the flux tubes less important.

The previous findings can explain the differences between stellarator and tokamaks previously observed in Fig. 1. In particular, the ASDEX Upgrade case [see Fig. 3(b)] reveals a more pronounced $\mathcal{K}_{y}$ negative contribution compared to W7-X, hence moving the optimal fast ion stabilizing temperature to smaller values. On the other side, in stellarator devices each field line is optimally stabilized by a different hydrogen temperature, leading to a broadening in the range of $T_{f} / T_{e}$ where the bulk ion energy flux is minimized.
$\mathcal{K}_{y}$ optimization.-The key role played by $\mathcal{K}_{y}$ in regulating the interaction between fast particles and turbulence makes this energetic particle mechanism potentially highly attractive for stellarators. Such devices typically possess $\sim 50$ degrees of freedom in the shaping of magnetic surfaces [24], which are here explored by designing artificial $\mathcal{K}_{y}$ able to enhance the wave-particle turbulence suppression. The simplest (two free parameters) parametrization of $\mathcal{K}_{y}$ able to reproduce the nominal W7-X and ASDEX Upgrade shape qualitatively is defined as

$$
\mathcal{K}_{y}=\mathcal{A} \frac{\sin (\mathcal{W} z-\pi / 2)}{1+0.15|z|}-0.02
$$

Here, $\mathcal{A}$ and $\mathcal{W}$ represent, respectively, the amplitude and the inverse width of $\mathcal{K}_{y}$. The dependence of the ITG microinstability with the changes in $\mathcal{K}_{y}$ is illustrated in Fig. 5 for $k_{y} \rho_{s}=0.5$ (i.e., the mode driving most of the turbulence in the simulations without fast ions), by performing hundreds of linear simulations for the bean-shaped flux tube without and with fast particles at $T_{f} / T_{e}=5$ (where the changes in the structure of $\mathcal{K}_{y}$ are more relevant). The remaining geometric coefficients are kept fixed to isolate its theoretical effect. The choice of this numerical setup is motivated by the bean-shaped flux tube being the most unstable field line [see Fig. 3(a)]. Therefore, its optimization is expected to have the strongest impact on the transport levels of the more computationally expensive full flux-surface simulations (for which a full scan would be unfeasible). In addition, a stronger stabilization at $T_{f} / T_{e}=5$ will also make the wave-particle resonance effect more accessible experimentally.

In the absence of energetic particles, we observe a detrimental effect of $\mathcal{A}$ on the ITG growth rates, which increase by a factor of 2 from small (W7-X-like) to large (ASDEX Upgrade-like) values. This result is consistent with the enhancement of the bad-curvature region (identified by the location where the magnetic curvature

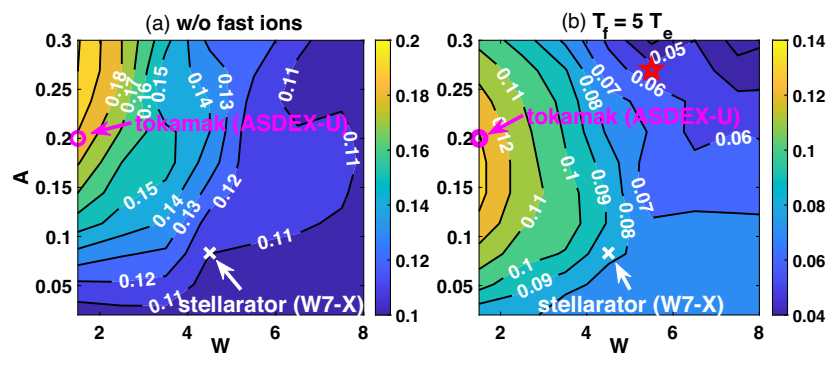

FIG. 5. Growth rates normalized to $c_{s} / a$ for different values of the amplitude $\mathcal{A}$ and inverse width $\mathcal{W}$ used in the $\mathcal{K}_{y}$ parametrization, obtained for the cases (a) without and (b) with energetic particles at $T_{f} / T_{e}=5$ for the bean-shaped flux tube. The red star denotes the optimized $\mathcal{K}_{y}$ configuration selected for the nonlinear analysis $(\mathcal{A}, \mathcal{W})=(0.27,5.5)$. 

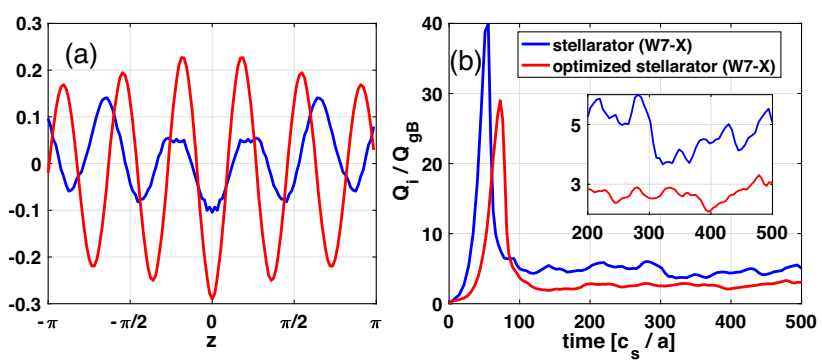

FIG. 6. Comparison of the (a) field-aligned structure of $\mathcal{K}_{y}$ and (b) time trace on the bulk ion heat flux for the nominal (blue line) and optimized (red line) W7-X geometry for the bean-shaped field line at $T_{f} / T_{e}=5$.

vector is parallel to the pressure gradient) with $\mathcal{A}$, thus enforcing the ITG drive term. Furthermore, Fig. 5(a) highlights the key role played by the inverse width $\mathcal{W}$. Its increase leads to more localized negative regions in $\mathcal{K}_{y}$, thus limiting the bad-curvature region able to drive ITG turbulence.

Interestingly, while the inclusion of energetic particles does not change the effect of $\mathcal{W}$ on the ITGs, it strongly affects the role of $\mathcal{A}$ by modifying the energetic particles drift frequency $\left(\omega_{d, f}\right)$. More precisely, an increase in $\mathcal{A}$ leads to a larger contribution of $\mathcal{K}_{y}$ in $\omega_{d, f}$, thus moving the resonance condition to smaller fast-particle temperatures (considering small changes in the ITG frequency) and enhancing the ITG stabilization at $T_{f} / T_{e}=5$. Therefore, the overall effect of $\mathcal{A}$ on the ITGs, shown in Fig. 5(b) for $T_{f} / T_{e}=5$, is the result of the competition between a detrimental (independent from the fast particles) and a stabilizing effect (due to the wave-particle interaction).

An optimized region with reduced ITG growth rates is identified in Fig. 5(b) (red star), with an additional stabilization of $35 \%$ compared to the nominal W7-X geometry. A comparison between the (W7-X) nominal and optimized stellarator $\mathcal{K}_{y}$ coefficients is displayed in Fig. 6(a). These results are confirmed by nonlinear simulations, where a $40 \%$ ITG turbulence suppression is measured in Fig. 6(b) by employing the optimized $\mathcal{K}_{y}$ configuration.

These findings provide evidence that suprathermal ions might strongly affect how to optimize future stellarator geometries. While in the absence of energetic particle, an improved turbulence stabilization requires a reduction in the amplitude of the curvature term $\mathcal{K}_{y}$, the opposite occurs in their presence. Therefore, configuration optimization codes such as STELLOPT [25] might be extended to capture the wave-particle resonance mechanism in the linear dispersion relation used as the proxy for the heat fluxes [26].

Conclusions. - In the present Letter, we show that suprathermal ions-e.g., from ICRF heating - can strongly suppress turbulent transport in optimized stellarators. This is demonstrated via state-of-the-art gyrokinetic turbulence simulations for W7-X. We find that the key mechanism for this suppression is the resonant waveparticle interaction between fast ions and ITG modes. This is the first time that such a mechanism is being proposed for present-day optimized stellarators. Therefore, these findings strongly motivate the use of ICRF heating systems in such devices as a means of reducing ion-scale core turbulent transport. We also identify stellaratorspecific features that impact the dynamics of the resonant interaction in these devices and discuss key differences compared to tokamaks. Moreover, we provide new insights into alternative stellarator magnetic configurations appositely designed to maximize the wave-particle resonance interaction. These results have important implications for the future optimization of such devices, where this resonant mechanism may be exploited systematically to strongly suppress particle and energy losses and hence access new improved scenarios with reduced transport levels.

The authors would like to thank G. G. Plunk, J. P. Martin Collar, T. Görler, E. Poli, and R. Bilato for stimulating discussions, useful suggestions, and comments. Numerical simulations were performed at the MARCONI- Fusion supercomputer at CINECA, Italy and at Cobra HPC system at the Max Planck Computing and Data Facility (MPCDF), Germany.

*These authors contributed equally to this work. †alessandro.disiena@austin.utexas.edu *alejandro.banon.navarro@ipp.mpg.de ${ }^{\S}$ frank.jenko@ipp.mpg.de

[1] U. Stroth, Plasma Phys. Controlled Fusion 40, 9 (1998).

[2] O. Motojima, N. Ohyabu, A. Komori, O. Kaneko, H. Yamada, K. Kawahata, Y. Nakamura, K. Ida, T. Akiyama, N. Ashikawa et al., Nucl. Fusion 43, 1674 (2003).

[3] C. Deng, D. Brower, D. Anderson, F. Anderson, A. Briesemeister, and K. Likin, Nucl. Fusion 55, 123003 (2015).

[4] P. Xanthopoulos, F. Merz, T. Görler, and F. Jenko, Phys. Rev. Lett. 99, 035002 (2007).

[5] P. Xanthopoulos, H. E. Mynick, P. Helander, Y. Turkin, G. G. Plunk, F. Jenko, T. Görler, D. Told, T. Bird, and J. H. E. Proll, Phys. Rev. Lett. 113, 155001 (2014).

[6] J. Nührenberg and R. Zille, Phys. Lett. 114A, 129 (1986).

[7] T. Klinger, C. Baylard, C. Beidler, J. Boscary, H. Bosch, A. Dinklage, D. Hartmann, P. Helander, H. Maberg, A. Peacock, T. Pedersen, T. Rummel, F. Schauer, L. Wegener, and R. Wolf, Fusion Eng. Des. 88, 461 (2013).

[8] F. Romanelli, Phys. Fluids B 1, 1018 (1989).

[9] R. Wolf, A. Ali, A. Alonso, J. Baldzuhn, C. Beidler, M. Beurskens, C. Biedermann, H.-S. Bosch, S. Bozhenkov, R. Brakel, A. Dinklage, Y. Feng, G. Fuchert, J. Geiger, O. Grulke, P. Helander et al., Nucl. Fusion 57, 102020 (2017).

[10] T. Klinger, T. Andreeva, S. Bozhenkov, C. Brandt, R. Burhenn, B. Buttenschön, G. Fuchert, B. Geiger, O. Grulke, H. Laqua et al., Nucl. Fusion 59, 112004 (2019). 
[11] N. Bonanomi, P. Mantica, A. Di Siena, E. Delabie, C. Giroud, T. Johnson, E. Lerche, S. Menmuir, M. Tsalas, D. Van Eester, and (JET Contributors), Nucl. Fusion 58, 056025 (2018).

[12] A. Di Siena, T. Görler, H. Doerk, E. Poli, and R. Bilato, Nucl. Fusion 58, 054002 (2018).

[13] A. Di Siena, T. Görler, E. Poli, R. Bilato, H. Doerk, and A. Zocco, Phys. Plasmas 26, 052504 (2019).

[14] A. J. Brizard and T. S. Hahm, Rev. Mod. Phys. 79, 421 (2007).

[15] M. Brambilla and R. Bilato, Nucl. Fusion 46, S387 (2006).

[16] J. Ongena, A. Messiaen, Y. Kazakov, B. Schweer, I. Stepanov, M. Vervier, M. Berte, K. Crombé, P. Despotin, F. Durodié, G. Jouniaux, A. Krivska, F. Louche, A. Lyssoivan, R. Philips, M. Van Schoor, and T. Wauters, in Proceedings of the 27th IAEA Fusion Energy Conference, (2018), Vol. IAEA CN-258, p. 7.

[17] F. Jenko, W. Dorland, M. Kotschenreuther, and B. N. Rogers, Phys. Plasmas 7, 1904 (2000).
[18] T. Görler, X. Lapillonne, S. Brunner, T. Dannert, F. Jenko, F. Merz, and D. Told, J. Comput. Phys. 230, 7053 (2011).

[19] M. Maurer, A. Bañón Navarro, T. Dannert, M. Restelli, F. Hindenlang, T. Görler, D. Told, D. Jarema, G. Merlo, and F. Jenko, J. Comput. Phys. 420, 109694 (2020).

[20] A. D. Siena, T. Görler, E. Poli, A. B. Navarro, A. Biancalani, and F. Jenko, Nucl. Fusion 59, 124001 (2019).

[21] A. Di Siena, Ph.D. thesis, Ulm University, Germany, 2019.

[22] H. Doerk, A. Bock, A. Di Siena, E. Fable, T. Görler, F. Jenko, J. Stober, and (The ASDEX Upgrade Team), Nucl. Fusion 58, 016044 (2018).

[23] Y. Suzuki and J. Geiger, Plasma Phys. Controlled Fusion 58, 064004 (2016).

[24] A. H. Boozer, Rev. Mod. Phys. 76, 1071 (2005).

[25] A. Reiman et al., Plasma Phys. Controlled Fusion 41, B273 (1999).

[26] H. E. Mynick, N. Pomphrey, and P. Xanthopoulos, Phys. Rev. Lett. 105, 095004 (2010). 